\title{
False negative hepatitis B surface antigen detection in dialysis patients due to excess surface antigen: postzone phenomenon
}

\author{
LG HEFTER, MARY A HIX, MICHELlE STONER, AND CB COOK \\ From The Fairfax Hospital, George Washington University School of Medicine, Washington, DC, USA
}

SUMMARY Renal dialysis patients are well known to have a high incidence of hepatitis B carrier state. In studying a group of 63 long-term dialysis patients, 10 were found to be positive for hepatitis B surface antigen by radioimmunoassay (RIA). Surprisingly, however, only three of these RIA positive patients were positive by counter immunoelectrophoresis (CIEP). The discrepancy could not be accounted for by the difference in sensitivity of the two methods. The cause for the negative reactions by CIEP in seven patients was found to be the marked excess surface antigen in these sera which produced false negative results by the postzone phenomenon. After dilution all seven sera were positive by CIEP, requiring a dilution up to $1 / 20$ to produce a positive result. Also, all seven sera were positive by the less sensitive Ouchterlony double diffusion.

Since the discovery of the Australian antigen by Blumberg et al. in 1965, ${ }^{1}$ its subsequent association with hepatitis $B$, and eventual identification as the protein surface coat of hepatitis B virus (HBsAg), there has been a search for more sensitive tests. These tests are now divided into first, second, and third generation types in order of increasing sensitivity. Ouchterlony double diffusion was the earliest test and is termed a first generation test. Counter immunoelectrophoresis (CIEP) is regarded as a second generation test in contrast to third generation tests such as radioimmunoassay (RIA) which are more sensitive. ${ }^{2}$ In studying a group of 63 dialysis patients, we happened to do CIEP in addition to RIA with some surprising results. Of 10 RIA positive patients only three were positive by CIEP. This marked discrepancy was not expected by the relative sensitivities of these two procedures and has not been our experience in the past when both procedures were performed on sera of blood donors. Many reports have dealt with the high incidence of hepatitis and hepatitis carrier states in dialysis patients. ${ }^{3}{ }^{4}$ One likely explanation for this is the immune deficiency found in uraemia. ${ }^{5} \mathrm{~A}$ corrolary of the deficient immune state and decreased cellular reaction within the liver is increased production of virus and surface antigen. Since the markedly reduced ability of CIEP to detect the RIA

Received for publication 19 February 1980 positive patients cannot be readily explained by the greater sensitivity of RIA, the possibility of excess $\mathrm{HBsAg}$ and subsequent postzone reaction was entertained. To test this hypothesis, CIEP was repeated after dilution of the sera. Also performed was Ouchterlony double diffusion in agarose.

\section{Patients and methods}

A group of 63 long-term haemodialysis patients, ranging in age from 21 to 60 , were tested for HBsAg by RIA and 10 were found to be positive. Sera from these patients were also tested for HBsAg by CIEP. Those that were negative by CIEP using undiluted serum were again run after dilution to $1 / 10$ and $1 / 20$ and were also tested by Ouchterlony double diffusion.

\section{RADIOIMMUNOASSAY}

The hepatitis B surface antigen was measured by RIA according to the manufacturer's instructions (Ausria II, Abbott Laboratories, Inc, Chicago, Illinois, USA).

\section{COUNTER IMMUNOELECTROPHORESIS}

The Hapindex method of Ortho Diagnostics was used according to the manufacturer's instructions. If results were negative or equivocal, they were repeated after dilution.

OUCHTERLONY DOUBLE DIFFUSION

Ouchterlony plates consisted of $1 \%$ agarose in 
phosphate buffer, pH 8.0 (Meloy Laboratories, Springfield, Virginia 22151, USA). The wells were $5 \mathrm{~mm}$ in diameter and $3 \mathrm{~mm}$ apart. Anti-HBsAg was placed in the centre well, and the patients' undiluted sera were placed in the surrounding wells. This antibody was that obtained with the Hapindex CIEP kit from Ortho Diagnostics. They were incubated in a moist chamber at room temperature and read at 24,48 , and 72 hours.

\section{Results}

Ten of 63 haemodialysis patients were positive for HBsAg by RIA but only three of these were clearly positive by CIEP using undiluted serum. Hazy zones near the antibody wells were found in six of the RIA positive sera. However, these were not distinct and could not be interpreted as positive. No such reaction was present in the remaining sample, which was totally negative. These seven sera were then serially diluted before CIEP testing. Positive reactions occurred in the sera of all after dilution. The highest dilution necessary to produce a positive reaction was $1 / 20$. An example is seen in the Figure

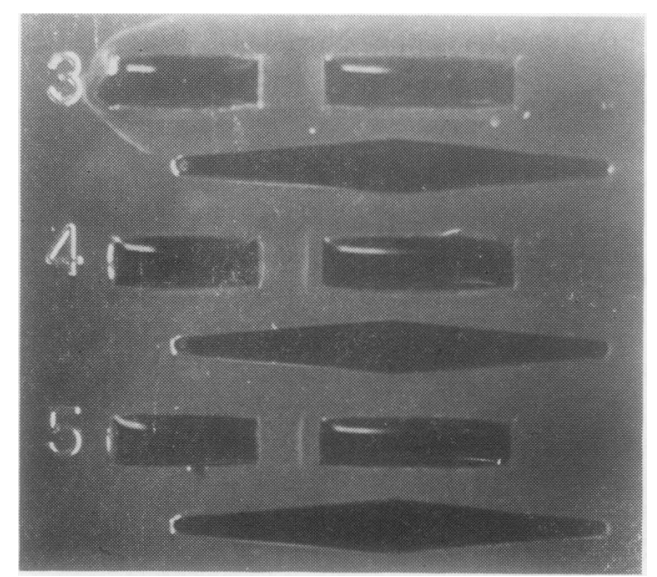

No precipitin band with undiluted serum (well 3) but distinct bands with serum diluted 1/10 and 1/20 (wells 4 and 5).

where the undiluted serum shows no precipitin band. At a dilution of $1 / 10$, however, there is a distinct band between the antigen-antibody wells. The band moves closer to the centre with a greater dilution of serum (1/20). All seven originally negative CIEP samples were positive by double diffusion.

\section{Discussion}

Third generation tests are advised for hepatitis B testing. However, in some areas of the world it is probable that CIEP is still being used, and it has been used to confirm RIA positive sera. The results of our studies show that the sera of $\mathrm{HBs} \mathrm{Ag}$ positive dialysis patients often contain a great quantity of surface antigen. This excess antigen produced false negative CIEP reactions by the postzone phenomenon in seven of 10 RIA positive dialysis patients. In contrast, all seven sera were positive by double diffusion, which is a much less sensitive test. The hypothesis of postzone reaction was proved when these became CIEP positive after dilution. This is a serious problem with CIEP which has not yet been emphasised. It can be obviated by dilution of the serum. CIEP negative results should be repeated using diluted sera in any patient known to be on dialysis, immunodeficient, or otherwise suspected of having hepatitis B infection. However, because of the lower sensitivity of CIEP in addition to the problem of postzone reaction, third generation tests such as RIA are advisable for all hepatitis testing.

\section{References}

${ }^{1}$ Blumberg BS, Alter HJ, Visnich S. A "new" antigen in leukemia sera. JAMA 1965;191:541-6.

${ }^{2}$ Chiaramonte M, Heathcote J, Crees M, Sherlock S. Detection by three techniques, of hepatitis B surface antigen (HBsAg) and determination of $\mathrm{HBsAg}$ and anti-HBs titres in patients with chronic liver disease. Gut 1977;18:1-6.

${ }^{3}$ Forrest JN, Dismukes WF. Dialysis associated hepatitis on 108 United States hemodialysis units. Clin Res 1969;16: 383.

${ }^{4}$ Hepatitis surveillance report 33. Atlanta Center for Disease Control. Public Health Service 1971.

${ }^{5}$ Boulton-Jones JM, Vick R, Cameron JS, Black PJ. Immune responses in uremia. Clin Nephrol 1973;1:351-60.

Requests for reprints to: Dr LG Hefter, The Fairfax Hospital, 3300 Gallows Road, Falls Church, Virginia 22046, USA. 